\title{
Electronic Power Conversion System for an Advanced Mobile Generator Set
}

\author{
Leon M. Tolbert ${ }^{1,3}$, William A. Peterson ${ }^{2}$, Matthew B. Scudiere ${ }^{3}$, Cliff P. White ${ }^{3}$, Timothy J. Theiss ${ }^{3}$, \\ John B. Andriulli ${ }^{3}$, Curt W. Ayers ${ }^{3}$, George Farquharson ${ }^{3}$, George W. Ott ${ }^{3}$, Larry E. Seiber ${ }^{3}$
}

${ }^{1}$ The University of Tennessee

Electrical and Computer Engineering

Knoxville, TN 37996-2100

Tel: (865) 974-2881

FAX: (865) 974-5483

e-mail: tolbert@utk.edu
${ }^{2}$ E\&M Power

6 Emma Street

Binghamton, NY 13905

Tel: (607) 766-9625

Fax: (607) 766-9622

peterson@EandMpower.com
${ }^{3}$ Oak Ridge National Laboratory

National Transportation Research Center

2360 Cherahala Blvd.

Knoxville, TN 37932

Tel: (865) 946-1328

Fax: (865) 946-1262

\begin{abstract}
The electronic power conversion system and control for an advanced mobile generator set is described. The military generator set uses an internal combustion diesel engine to drive a radial-gap permanent magnet alternator at variable speed. The speed of the engine is determined from a user selectable interface that for a given load and ambient thermal conditions controls the engine to run at its most efficient operating point. It is also possible to control the engine to run where it is most audibly quiet, at its least-polluting operating point, or at its most reliable, stiffest point such that it is less sensitive to load transients. The variable frequency, variable voltage produced by the permanent magnet alternator is diode-rectified to dc voltage, and an inverter is used to produce selectable frequency, controllable ac voltage. The power conversion system also incorporates a bi-directional dc-dc converter that can charge $24 \mathrm{~V}$ batteries that are used to start the IC engine and to power auxiliary loads. The bi-directional converter can also draw power from the batteries to help maintain the dc link during severe load transients. The design of this new generator set offers additional flexibility by being lighter, smaller, and more fuel efficient than a conventional fixed-speed gen-set.
\end{abstract}

\section{INTRODUCTION}

The U.S. Department of Defense has initiated a program to upgrade and develop enhanced tactical power systems to provide reliable electrical power in the battlefield. One aspect of this program is for the development of advanced mobile generator sets in the medium power $(5-60 \mathrm{~kW})$ range. These future generator sets are to be portable, lightweight systems that are electronically controlled, signature suppressed, and capable of operating on DF-2/JP-8 fuels in extreme environmental conditions. This paper describes a proof of concept development of a military gen-set in the medium power range.

Existing military gen-sets in the medium power range have been designed to be extremely rugged, cost-effective sources of power. These units are significantly heavier than their industrial counterparts to meet the stringent ruggedness requirements of the military and because the units provide additional features and capabilities that are not available on industrial units. Military mobile electric generators are designed to operate in a temperature range from $-45^{\circ} \mathrm{C}$ to $60^{\circ} \mathrm{C}$ and at altitudes ranging from sea level to $3000 \mathrm{~m}$.

Existing military gen-sets have a diesel combustion engine that is governed to run at a fixed speed such that the directly coupled alternator driven by the engine produces a fixed frequency output of either 50 or $60 \mathrm{~Hz}$ in some units or 400 $\mathrm{Hz}$ in other units. Brushless synchronous machines are presently used to convert the mechanical power of the rotating shaft into three-phase electrical voltage at a preset frequency.

In order to reduce their logistics burdens, the DOD would like future gen-sets to be much lighter, smaller in volume, more fuel-efficient, quieter, and more reliable than the existing units. Fixed-speed engines are forced to run outside their optimum fuel consumption envelope when these units are not run near full load; therefore, running the engine at variable speed and using power electronics to convert the variable voltage and frequency to a fixed voltage and frequency can allow more efficient operation of the gen-set [1-6].

A block diagram of the electronic power conversion system for the proof of concept generator set is shown in Fig. 1. The military generator set uses an internal combustion diesel engine to drive a radial-gap permanent magnet alternator at variable speed. With advanced diesel engines and variable speed technology, goals for the program were to reduce weight by almost $50 \%$ and increase efficiency by up to $30 \%$. The speed of the engine is determined from a user selectable interface that for a given load and ambient thermal conditions allows the engine to run at its most efficient operating point. It is also possible to control the engine to run where it is most audibly quiet, its least-polluting operating point (from an emissions point of view), or at its most reliable, stiffest point such that it is less sensitive to load transients.

The variable frequency, variable voltage produced by the permanent magnet alternator is diode-rectified to dc voltage, 


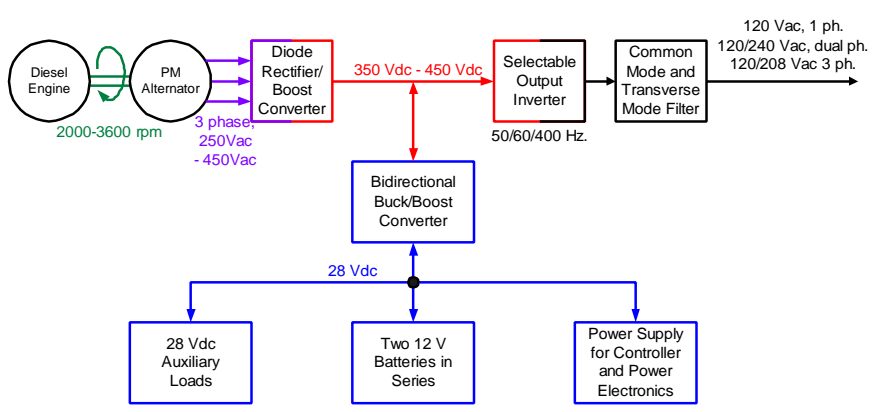

Fig.1. Functional block diagram of power conversion components in generator set.

and an inverter is used to produce selectable frequency, controllable ac voltage. The user is allowed to select singlephase $120 \mathrm{~V}$, dual-phase $120 / 240 \mathrm{~V}$, or three-phase $120 / 208 \mathrm{~V}$. Each of these voltage configurations can be generated at $50 \mathrm{~Hz}, 60 \mathrm{~Hz}$, and $400 \mathrm{~Hz}$ such that the unit can be compatible with equipment produced from around the world or for aerospace applications. The power conversion system also incorporates a bi-directional dc-dc converter that can charge 24V batteries that are used to start the IC engine and to power auxiliary loads. The converter can also draw power from the batteries to help maintain the dc link during severe load transients. The design of this new generator set offers additional flexibility by being lighter, smaller, and more fuel efficient than conventional fixed-speed gen-sets.

\section{ENGINE AND ALTERNATOR DESCRIPTION}

Each generator set size was determined by selecting an advanced diesel engine that had a high power to weight ratio. For the smallest gen-set, an air-cooled Ruggerini MD 191 rated at $13.0 \mathrm{~kW}$ was used as the prime mover. The engine was completely characterized for power, torque, fuel consumption, and emissions of $\mathrm{CO}_{2}, \mathrm{CO}, \mathrm{NOx}, \mathrm{CO}$, and THC over its operating speed range. Fig. 2 shows peak power, torque, and fuel consumption over the engine's operating range.

For each engine size, a radial gap permanent magnet

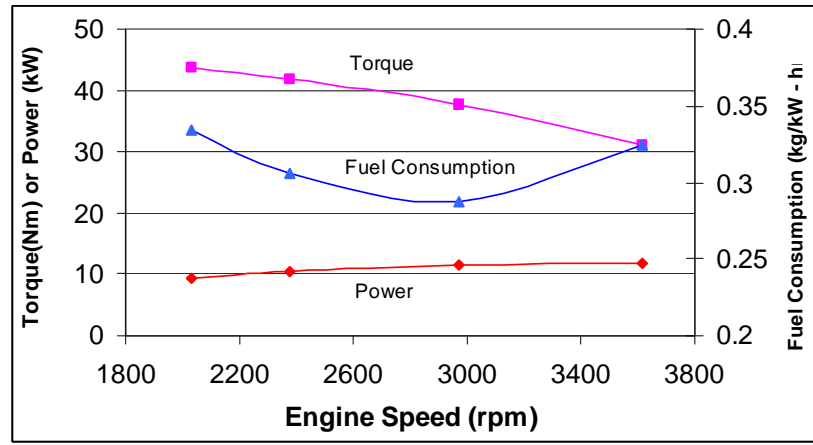

Fig. 2. Peak torque, power, and fuel consumption for the diesel engine in proof of concept gen-set. alternator with high flux magnets $(\mathrm{NeFeB})$ was designed such that the alternator produced trapezoidal back emf with a peak line-line voltage of $450 \mathrm{~V}$ at the top engine speed. This allowed enough design margin such that $600 \mathrm{Vdc}$ devices

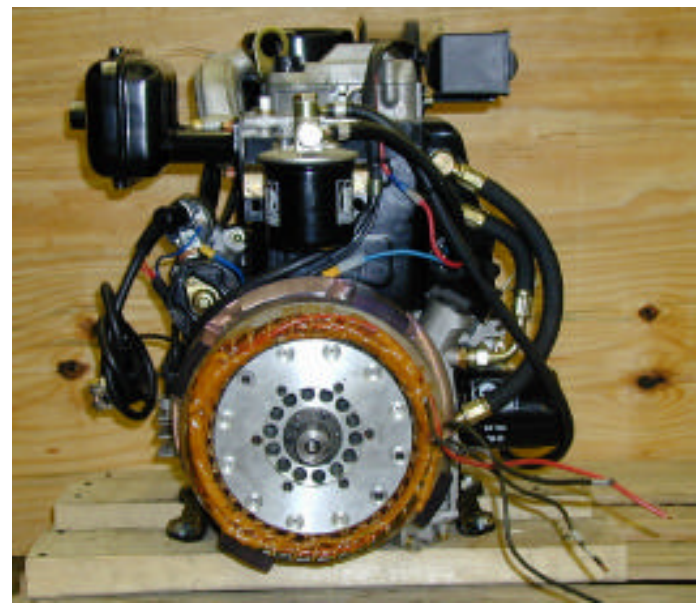

Fig. 3. PM alternator mounted to gen-set engine.

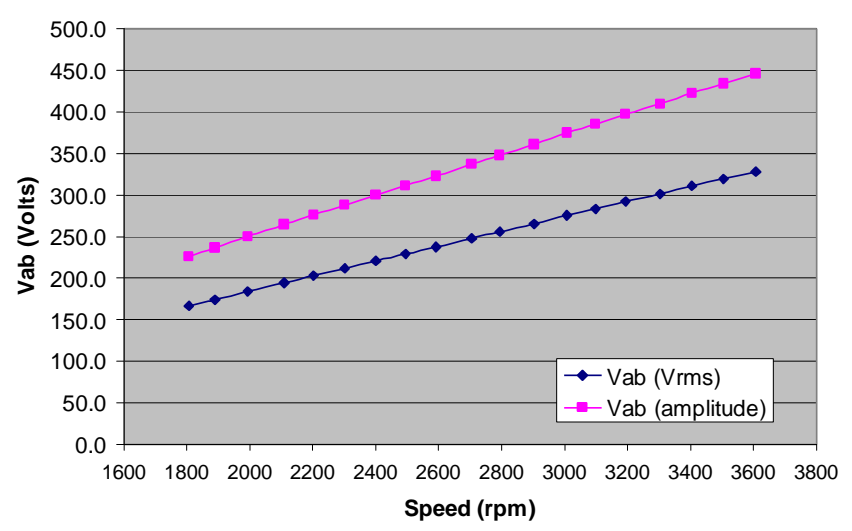

Fig. 4. PM Alternator voltage versus speed.

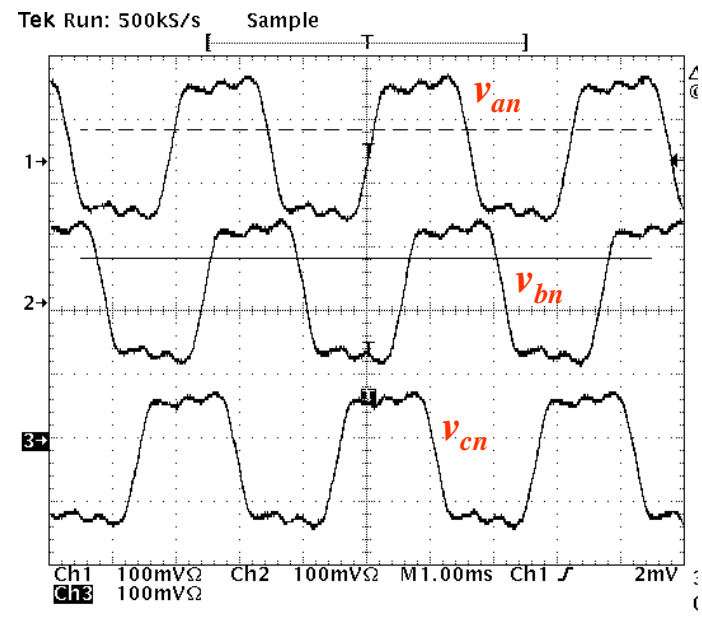

Fig. 5. PM Alternator voltage waveforms at $3200 \mathrm{rpm}$. 
were used for the main power components. A bearingless cantilever design for the alternator allowed the stator to bolt directly to the engine block. The rotor mounted directly to the engine shaft, and it had a diameter of $17 \mathrm{~cm}$ and a depth of $4 \mathrm{~cm}$. Fig. 3 shows the Ruggerini engine coupled to the PM alternator. Line-line peak output voltage of the PM alternator is shown in Fig. 4 to vary linearly with speed, as expected, such that at $3600 \mathrm{rpm}$ the alternator produced a waveform with a line-line amplitude of $450 \mathrm{~V}$, and at 2000 rpm a voltage waveform with an amplitude of $250 \mathrm{~V}$. Fig. 5 shows line-neutral voltage waveforms for the alternator operating at $3200 \mathrm{rpm}$.

\section{ASYNCHRONOUS BOOST OPERATION}

The gen-set was designed such that the inverter can produce 120 Vrms line-neutral voltage for dc link voltages between $350 \mathrm{Vdc}$ and $450 \mathrm{Vdc}$. During normal, unboosted operation, the alternator line-line voltage is rectified by the three-phase, full bridge rectifier $D_{1}$ with conventional six-pulse rectification as shown in Fig. 6. During this mode, the boost rectifier is inactive and transistor $\mathrm{Q}_{1}$ is off. The minimum desired dc link voltage of $350 \mathrm{Vdc}$ corresponds to an alternator speed of $2800 \mathrm{rpm}$.

When the DC link voltage produced by this normal mode of rectification is less than $350 \mathrm{Vdc}$ (corresponding to a gen-set speed of 2000 to $2800 \mathrm{rpm}$ ), the asynchronous boost circuit becomes active to maintain the dc link voltage. The circuit that boosts the alternator voltage when the speed is low operates asynchronously with rotor position. When the boost is active, transistor $\mathrm{Q}_{1}$ turns on and effectively shorts out the alternator through the full-bridge rectifier $\mathrm{D}_{2}$ shown in Fig. 6. During this time, the three-phase full-bridge rectifier diodes in $\mathrm{D}_{1}$ block, and capacitor $\mathrm{C}_{1}$ supplies the inverter load. The current being supplied by the alternator ramps up due to this short and is limited only by the alternator leakage inductance.

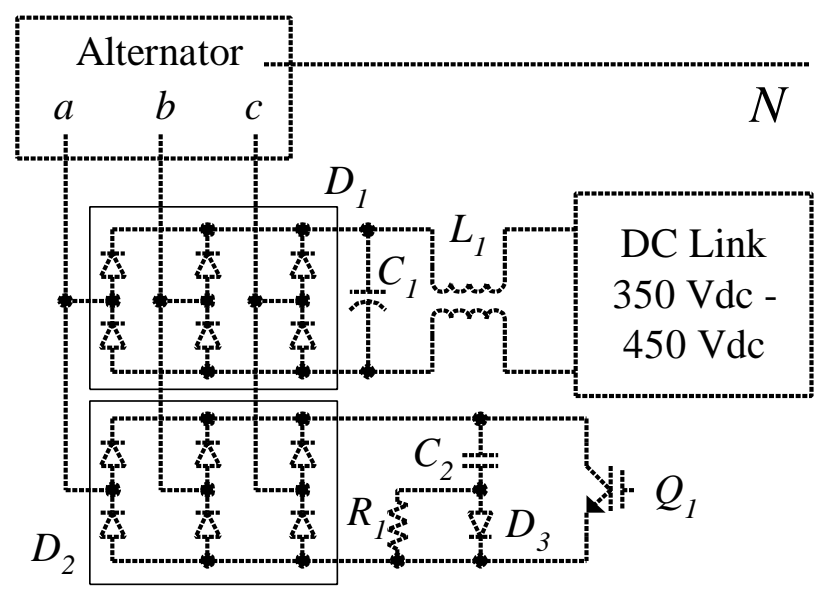

Fig. 6. Schematic of gen-set boost rectifier.
When $Q_{1}$ turns off, the current then flows through the threephase full-bridge rectifier $D_{1}$ to charge $C_{1}$. Transistor $Q_{1}$ is operated as a DC current mode boost, and the diodes in $\mathrm{D}_{1}$ must be the fast recovery type because they are operated at the boost switching frequency. The diodes in $\mathrm{D}_{2}$ are commutated at the alternator frequency and therefore do not have reverse recovery requirements. The parasitic inductance of the path through $\mathrm{D}_{1}$ and $\mathrm{C}_{1}$ where the boost current flows when $\mathrm{Q}_{1}$ is turned off can cause a large voltage spike at $\mathrm{Q}_{1}$. However, the snubber composed of $\mathrm{C}_{2}$ and $\mathrm{D}_{3}$ offers a low inductance diversion path for this current and thus limits the di/dt to a value that will not produce damaging voltage spikes.

The alternator neutral and load neutral are connected to the center of two capacitors $\mathrm{C}_{3}$ shown in Fig. 7. The connection allows unbalanced load currents to flow through the alternator in parallel with the capacitors and eliminates the requirement that they have a low impedance at the fundamental frequency. The inductor $L_{1}$ shown in Fig. 6 prevents what would be an undesired consequence of this connection. Without $\mathrm{L}_{1}$, each of the $\mathrm{C}_{3}$ capacitors would charge to the half-wave rectified line-neutral voltage of the alternator, which results in poor power factor and high crest factor in the alternating current.

\section{INVERTER AND OUTPUT FILTER DESCRIPTION}

A traditional full-bridge three-phase inverter composed of a $600 \mathrm{~V}, 200 \mathrm{~A}$ six-pack IGBT module $\mathrm{Q}_{2}$ (Eupec BSM200GD60LD) was controlled with a digital signal processor (DSP) to produce a PWM output as shown by the

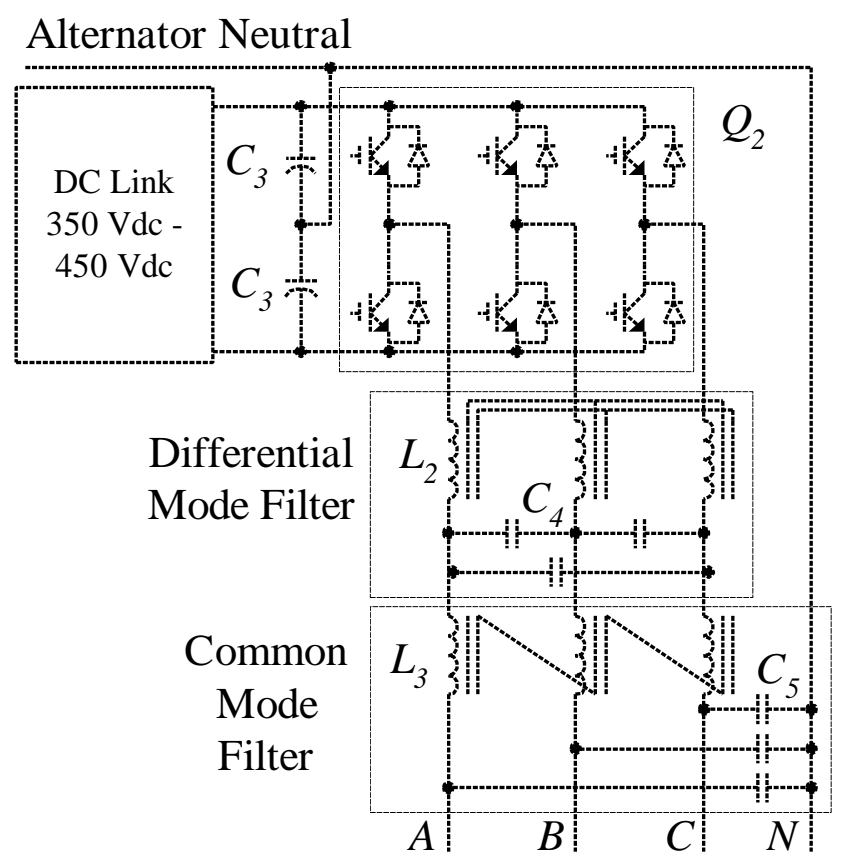

Fig. 7. Schematic of gen-set inverter and filters. 


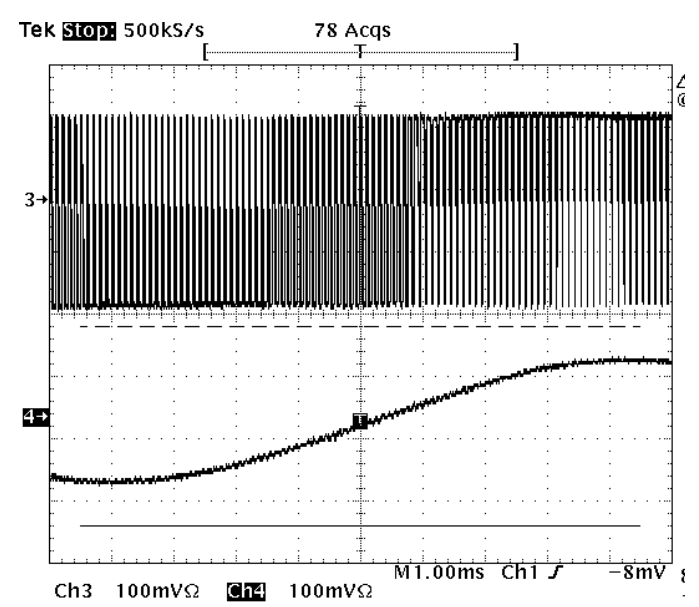

Fig. 8. Pre- and post-filtered line-neutral output voltage waveforms from the proof of concept gen-set.

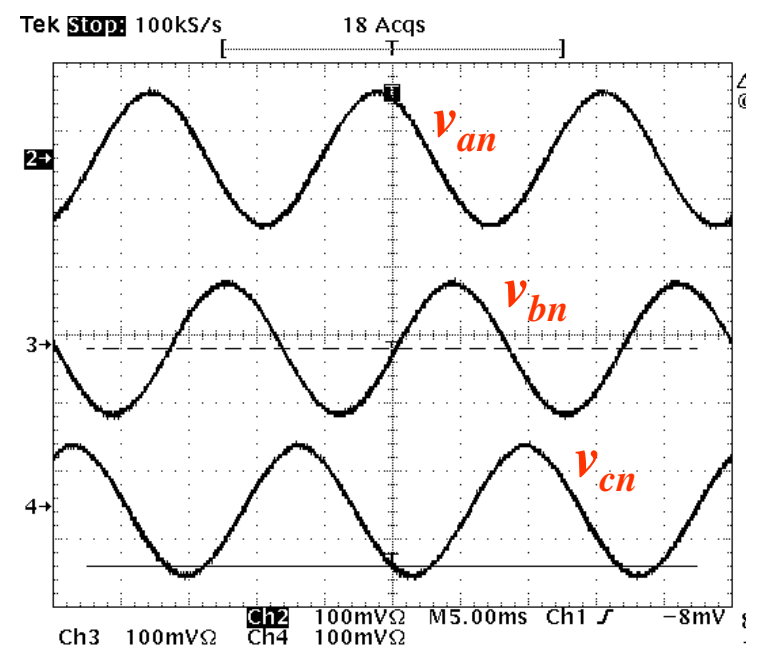

Fig. 9. Filtered line-neutral inverter output voltage waveforms.

schematic in Fig. 7. The PWM waveform is passed through a three-phase common-mode LC filter composed of $\mathrm{L}_{3}$ and $\mathrm{C}_{5}$ and a three-phase differential mode $\mathrm{LC}$ filter composed of $\mathrm{L}_{2}$ and $\mathrm{C}_{4}$ to achieve near sine-wave ( $<3 \%$ total harmonic distortion) waveforms as shown in Figs. 8 and 9. The differential mode filter was designed for a corner frequency of $3.3 \mathrm{kHz}$, and the common mode filter has a corner frequency of $1.3 \mathrm{kHz}$.

\section{Bi-Directional CONVERTER DESCRIPTION}

The bi-directional power supply takes power from the $350 \mathrm{~V}$ dc link and converts it to $28 \mathrm{~V}$ to power $28 \mathrm{~V}$ auxiliary loads and to recharge the engine starting battery. The converter is

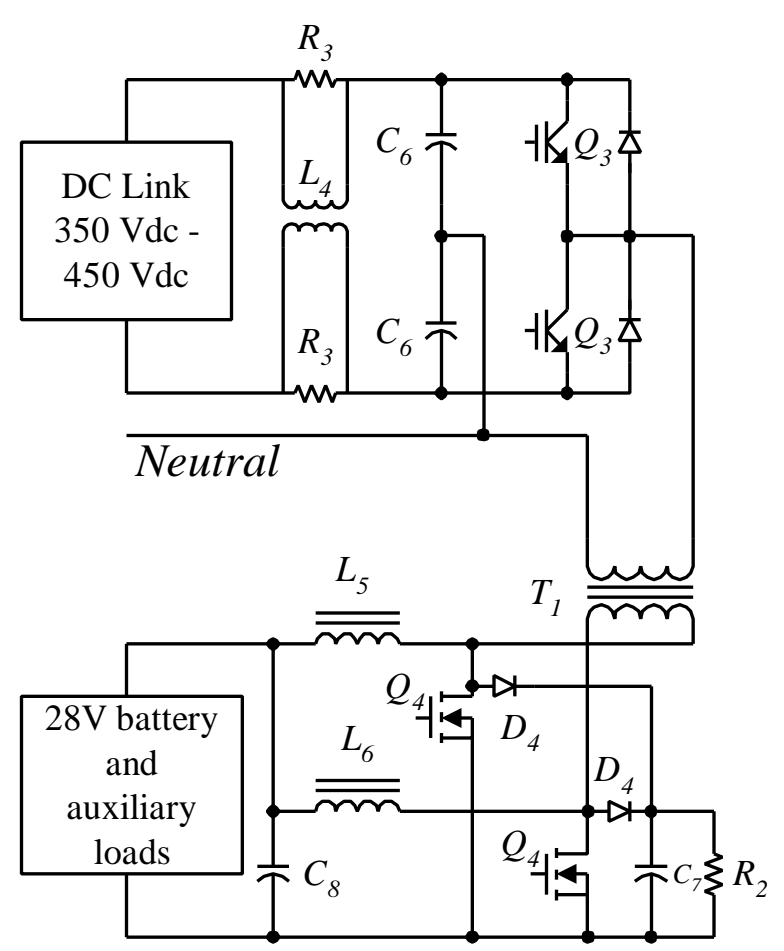

Fig. 10. Schematic of gen-set bi-directional converter.

able to provide $7 \mathrm{~kW}$ for less than 10 seconds or $1.4 \mathrm{~kW}$ continuously. When operating in this buck mode, the converter operates as a voltage mode half-bridge transformer coupled buck regulator with a current doubler rectifier. In boost mode, the converter can use the $28 \mathrm{~V}$ power from the battery to supply the dc link during load transients when the engine has not responded with sufficient speed to maintain the dc link above $350 \mathrm{Vdc}$. The selection of these two topologies was to minimize the energy storage requirements and transformer turns ratio such that it is of minimum size and weight yet able to provide enough ride-through until the engine can pick up the load.

For high voltage to low voltage operation, the dc link voltage is passed through an R-L link damper consisting of the two $R_{3}$ resistors and $L_{4}$ as shown in Fig. 10. This link damper prevents oscillation between the $\mathrm{C}_{6}$ capacitors of the bi-directional converter and the DC link capacitors $\mathrm{C}_{3}$ on the inverter (Fig. 7). The $\mathrm{C}_{6}$ capacitors serve as high frequency energy storage for power transfer in either direction and make up the reactive half of the half bridge converter with the $\mathrm{Q}_{3}$ IGBTs.

To minimize the size of the high frequency transformer $\mathrm{T}_{1}$, the $\mathrm{Q}_{3}$ IGBTs are driven at a $20 \mathrm{kHz}$ frequency. Transformer $\mathrm{T}_{1}$ has a turns ratio of $2.8: 1$; this combined with the voltage halving of the half-bridge and a second voltage halving by the current doubler rectifier (diodes $\mathrm{D}_{4}$ and filtered by $\mathrm{L}_{5}$ and $\mathrm{L}_{6}$ ) allows the $\mathrm{Q}_{3}$ IGBTs to be operated at a maximum duty ratio of 0.85 when converting $350 \mathrm{~V}$ to $30 \mathrm{~V}$. 


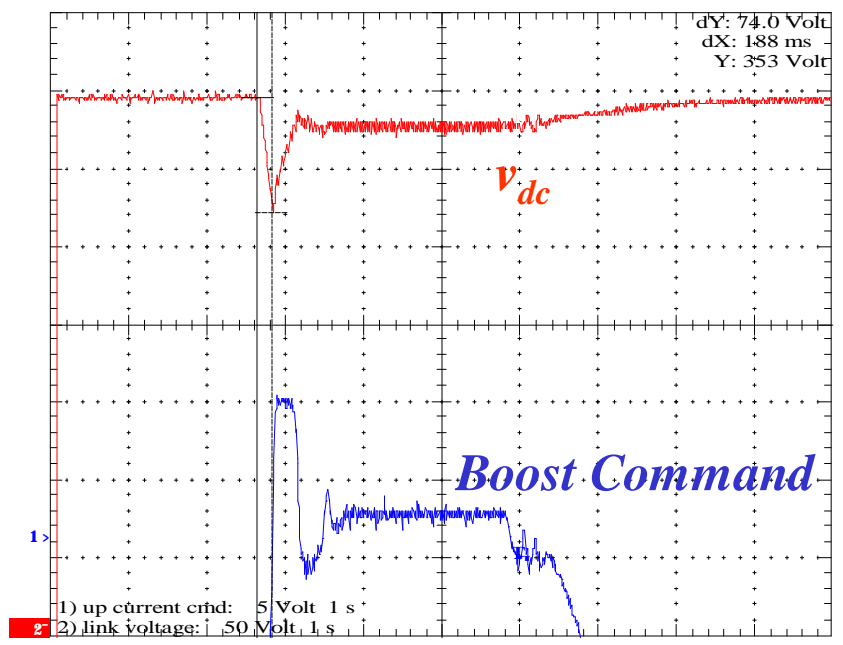

Fig. 11. DC link voltage maintained by boost from bi-directional converter upon loss of voltage from prime mover.

For low voltage to high voltage operation, the boost mode is activated when the dc link voltage falls to $340 \mathrm{Vdc}$, or $10 \mathrm{~V}$ below the nominal dc link voltage. When both of the $\mathrm{Q}_{4}$ transistors are on, transformer $\mathrm{T}_{1}$ is shorted and the battery voltage is applied to inductors $\mathrm{L}_{5}$ and $\mathrm{L}_{6}$. When one of the two switches is turned off, the input voltage plus the energy stored in the inductor is applied to $T_{1}$. Transformer $T_{1}$ steps up the voltage, which is then rectified by the antiparallel diodes of the $\mathrm{Q}_{3}$ IGBTs. When both of the $\mathrm{Q}_{4}$ switches are off, any stored energy in inductors $\mathrm{L}_{5}$ and $\mathrm{L}_{6}$ is transferred through $\mathrm{D}_{4}$ to $\mathrm{C}_{7}$, which is then discharged by $\mathrm{R}_{2}$.

Fig. 11 shows how the bi-directional converter is able to maintain the dc link voltage under the extreme case of complete loss of power from the rectified alternator voltage. The dc link voltage dips from $350 \mathrm{~V}$ to $280 \mathrm{~V}$ for the case shown in Fig. 11, but the converter will operate under the less stringent requirements of maintaining the dc link during a short-term overload and not for a complete loss of power from the prime mover.

\section{CONTROLS}

The 7.5kW-rated proof of concept gen-set is shown in Fig. 12. A user control and status display panel on the gen-set unit provides an interface to a digital control system. The controls were implemented on two TI TMS320F6701 floating point DSP processors of the Daytona dual DSP PCI card. In addition to this, a PCI interface card was designed and implemented to interface the DSP card via a 32-bit parallel port. This interface board (OrnlDas) contains eight independent 14-bit $\mathrm{A} / \mathrm{D}$ converters that operate at $1.2 \mu \mathrm{sec}$ and twelve more analog channel inputs that operate at $5 \mu \mathrm{sec}$. An OrnlDas card also contains a UART to communicate to

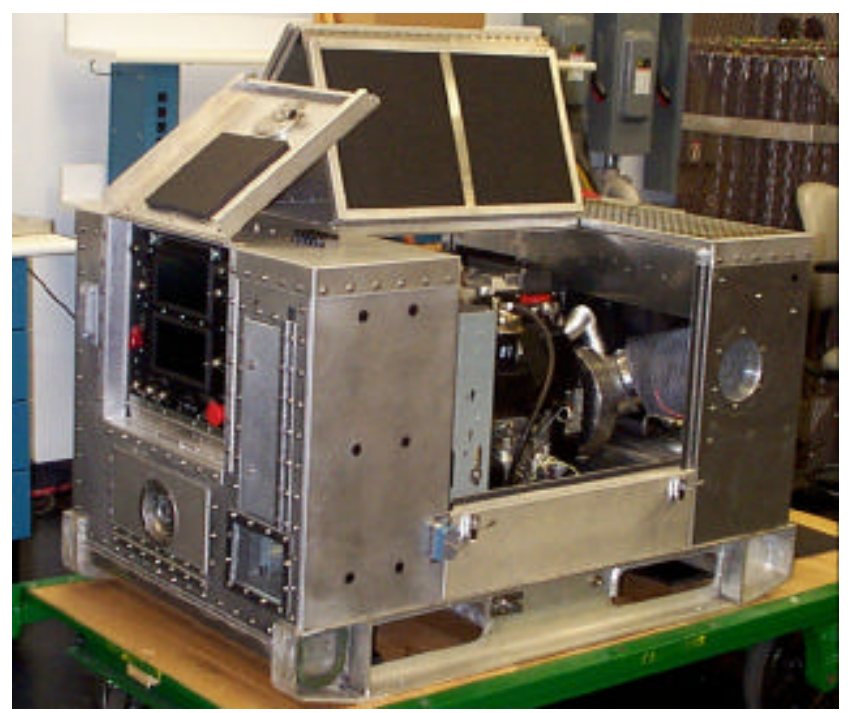

Fig.12. Proof of concept 7.5kW-rated gen-set.

the Front Panel displays, four 16 bit D/As for analog control, and four 16-bit input and output digital channels.

The control is split between the two DSPs on the Daytona card. The primary DSP, which can communicate with the OrnlDas card, performs all of the I/O with the rest of the genset. It also regulates the output voltage on a point by point basis. The second DSP takes the analog and digital data and computes RMS voltages and currents, power levels, and power factors of the AC waveforms; the dc link, battery, and $28 \mathrm{~V}$ auxiliary load voltages and currents; and the averages of temperatures, oil pressure, engine speed, etc. These are displayed on the screens on one or more of the display selections. Other functions of the DSPs include control of the output current to generate PWM duty cycles and the determination of the optimum engine speed for a given output power.

All operator inputs on the display panel, shown in Fig. 13, are routed directly to the DSP. It is the DSP that then interprets the information and decides on the "safe" conditions before controlling the rest of the system. Since it is the software that interprets the functions of all the switches and other inputs, it is easy to change or update the control logic to match different conditions, engines, or even power electronics. The system was also designed to enable the addition of diagnostics and prognostics to facilitate field maintenance and repair.

The display panel allows the user to monitor phase voltages, currents, power, and power factor from a user digital display. The display also shows the engine speed and the status of certain parameters such as oil pressure and temperature and intake or exhaust temperature. Output selection of $120 \mathrm{~V} /$ single phase, $240 \mathrm{~V} /$ dual phase, and 208 $\mathrm{V} /$ three phase are switch selectable as shown at the bottom of the picture in Fig. 13. The output frequencies of $50 \mathrm{~Hz}$, 
$60 \mathrm{~Hz}$, and $400 \mathrm{~Hz}$ are also switch selectable. Once the electrical output is energized, however, these switches are ignored to prevent false changes during operation and damage of the connected load.

\section{CONCLUSIONS}

Several power electronic converters have been implemented in an advanced mobile generator set such that its size, weight, and flexibility of voltage and frequency options is much improved over conventional fixed speed generator sets. A proof of concept unit has been implemented with experimental results shown for the converter components. With integrated controls and power electronics, the generator set is able to run at its most efficient condition to minimize fuel consumption. It would also be possible to control the gen-set such that it ran at other different optimized conditions: (1) maximum headroom for supplying load transients, (2) most audibly quiet, (3) and least polluting for certain emissions.

\section{ACKNOWLEDGMENTS}

This project is part of the Advanced Medium-Sized Mobile Power System (AMMPS) program conducted by the U.S. Army Communications \& Electronics Command (CECOM) Research, Development, \& Engineering Center, Power Generation Branch under the sponsorship of the Department of Defense Program Manager for Mobile Electric Power (PM-MEP).

\section{REFERENCES}

[1] M. J. Ryan, R. D. Lorenz, "A Power-Mapping Variable-Speed Control Technique for a Constant-Frequency Conversion System Powered by an IC Engine and PM Generator," IEEE IAS 2000 Annual Meeting, Rome, Italy, pp. 2376-2382.

[2] J. B. Andriulli, M. B. Scudiere, L. M. Tolbert, et al., "Advanced Power Generation Systems for the $21^{\text {st }}$ Century," ORNL/TM-1999/213, Oak Ridge National Laboratory, November 1999.

[3] H. Scott, C. Sun, K. Pandya, "Light Weight Portable Generator Set with Electrical Load Demand Governor," IEEE International Conference on Electric Machines and Drives, 1997.

[4] Z. Chen, E. Spooner, "Power Conversion System for a Modular, DirectDrive, Permanent-Magnet Wind Turbine Generator," IEE Colloqium on Power Electronic for Renewable Energy, June 1997, pp. 1-5.

[5] R. Krishnan, G. H. Rim, "Modeling, Simulation, and Analysis of VariableSpeed Constant Frequency Power Conversion Scheme with a Permanent Magnet Brushless DC Generator," IEEE Trans. Industrial Electronics, vol. 37, no. 4, Aug. 1990, pp. 291-296.

[6] R. Krishnan, G. H. Rim, "Performance and Design of a Variable Speed Constant Frequency Power Conversion Scheme with a Permanent Magnet Synchronous Generator," IEEE IAS 1989 Annual Meeting, pp. 45-50.

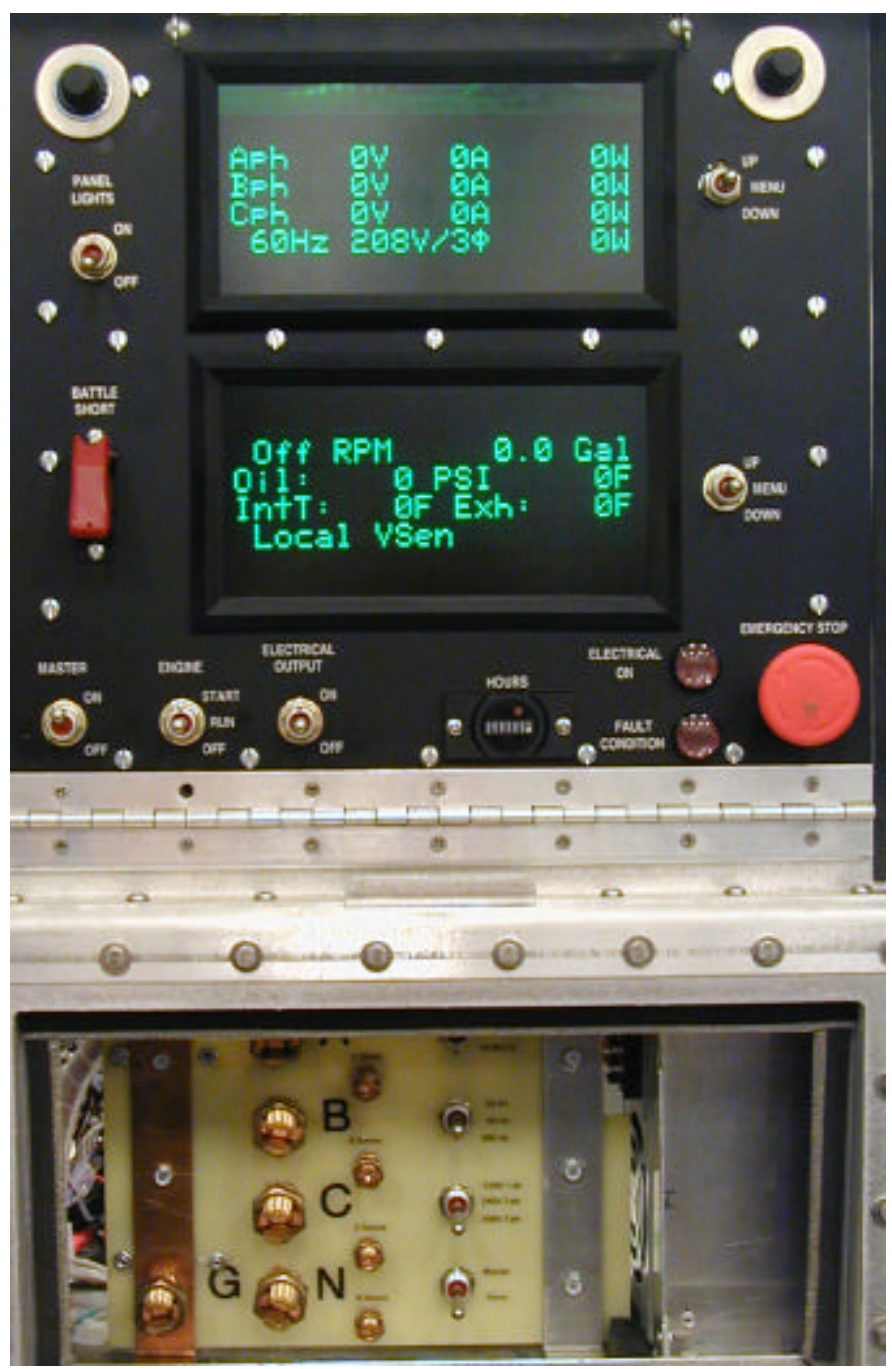

Fig.13. Gen-set user interface and display panel (top half of picture) and field output connections and voltage configuration and frequency selection switches (bottom half of picture). 